\title{
High-capacity hydrogen storage by metallized graphene
}

C. Ataca, E. Aktürk, S. Ciraci, and H. Ustunel

Citation: Appl. Phys. Lett. 93, 043123 (2008);

View online: https://doi.org/10.1063/1.2963976

View Table of Contents: http://aip.scitation.org/toc/apl/93/4

Published by the American Institute of Physics

\section{Articles you may be interested in}

Metal-dispersed porous graphene for hydrogen storage

Applied Physics Letters 98, 093103 (2011); 10.1063/1.3560468

Al doped graphene: A promising material for hydrogen storage at room temperature Journal of Applied Physics 105, 074307 (2009); 10.1063/1.3103327

Enhancement of hydrogen physisorption on graphene and carbon nanotubes by Li doping

The Journal of Chemical Physics 123, 204721 (2005); 10.1063/1.2125727

Density functional study of adsorption of molecular hydrogen on graphene layers

The Journal of Chemical Physics 112, 8114 (2000); 10.1063/1.481411

Strain effects on hydrogen storage capability of metal-decorated graphene: A first-principles study Applied Physics Letters 97, 103109 (2010); 10.1063/1.3486682

Hydrogen storage with titanium-functionalized graphene

Applied Physics Letters 103, 013903 (2013); 10.1063/1.4812830

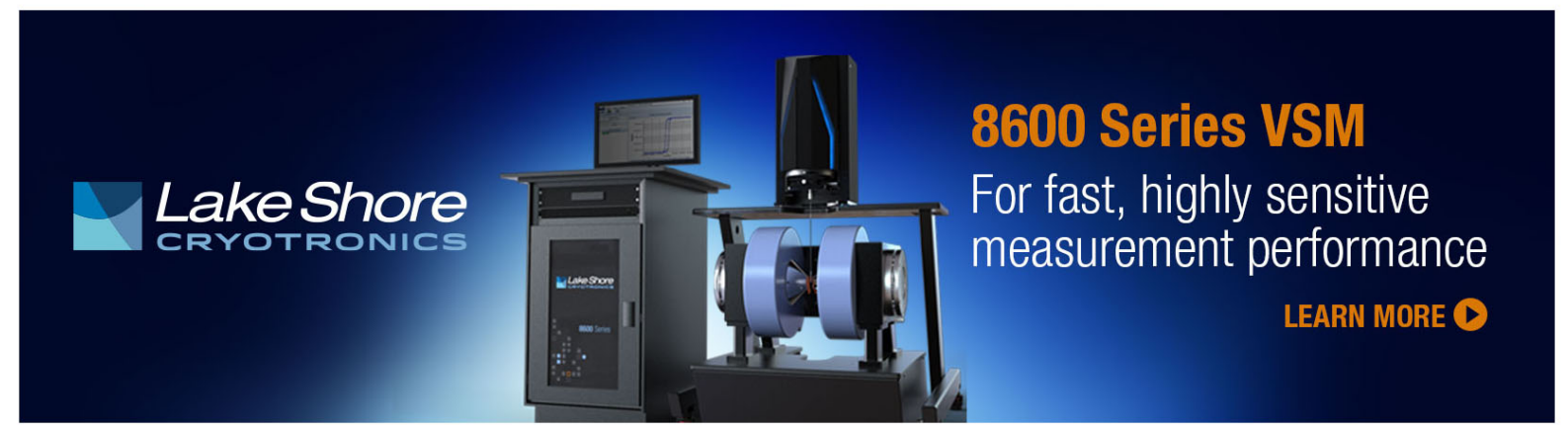




\title{
High-capacity hydrogen storage by metallized graphene
}

\author{
C. Ataca, ${ }^{1,2}$ E. Aktürk, ${ }^{2}$ S. Ciraci, ${ }^{1,2, a)}$ and H. Ustunel ${ }^{3}$ \\ ${ }^{1}$ Department of Physics, Bilkent University, Ankara 06800, Turkey \\ ${ }^{2}$ UNAM-Institute of Materials Science and Nanotechnology, Bilkent University, Ankara 06800, Turkey \\ ${ }^{3}$ Department of Physics, Middle East Technical University, Ankara 06531, Turkey
}

(Received 23 April 2008; accepted 3 July 2008; published online 31 July 2008)

\begin{abstract}
First-principles plane wave calculations predict that $\mathrm{Li}$ can be adsorbed on graphene forming a uniform and stable coverage on both sides. A significant part of the electronic charge of the Li $2 s$ orbital is donated to graphene and is accommodated by its distorted $\pi^{*}$-bands. As a result, semimetallic graphene and semiconducting graphene ribbons change into good metals. It is even more remarkable that $\mathrm{Li}$ covered graphene can serve as a high-capacity hydrogen storage medium with each adsorbed $\mathrm{Li}$ absorbing up to four $\mathrm{H}_{2}$ molecules amounting to a gravimetric density of 12.8 wt \%. (C) 2008 American Institute of Physics. [DOI: 10.1063/1.2963976]
\end{abstract}

Developing safe and efficient hydrogen storage is essential for hydrogen economy. ${ }^{1}$ Recently, much effort has been devoted to engineer carbon based nanostructures, ${ }^{2-5}$ which can absorb $\mathrm{H}_{2}$ molecules with high storage capacity, but can release them easily in the course of consumption in fuel cells. Insufficient storage capacity, slow kinetics, poor reversibility, and high dehydrogenation temperatures have been the main difficulties toward acceptable media for hydrogen storage.

Recently, graphene, a single atomic plane of graphite, has been produced ${ }^{6}$ showing unusual electronic and magnetic properties. In this letter, we predict that metallized graphene can be a potential high-capacity hydrogen storage medium. The process is achieved in two steps. Initially, graphene is metallized through charge donation by adsorbed $\mathrm{Li}$ atoms to its $\pi^{*}$-bands. Subsequently, each positively charged Li ion can absorb up to four $\mathrm{H}_{2}$ by polarizing these molecules. At the end, the storage capacity up to the gravimetric density of $g_{d}=12.8 \mathrm{wt} \%$ is attained. These results are important not only because graphene is found to be a high capacity hydrogen storage medium, but also because of its metallization through Li coverage is predicted.

Our results have been obtained by performing firstprinciples plane wave calculations using ultrasoft pseudopotentials. $^{7}$ We used local density approximation (LDA), since the van der Waals contribution to the Ligraphene interaction has been shown ${ }^{8}$ to be better accounted by LDA. Numerical results have been obtained by using VASP, ${ }^{9}$ which were confirmed by using the PWSCF code. ${ }^{10} \mathrm{~A}$ plane-wave basis set with kinetic energy cutoff $\hbar^{2} \mid \mathbf{k}$ $+\left.\mathbf{G}\right|^{2} / 2 m=380 \mathrm{eV}$ has been used. In the self-consistent potential and total energy calculations the Brillouin zone has been sampled by $(19 \times 19 \times 1)$ and $(9 \times 9 \times 1)$ special mesh points in $\mathbf{k}$ space for $(2 \times 2)$ and $(4 \times 4)$ graphene cells, respectively. Atomic positions in all structures are optimized using the conjugate gradient method. Convergence is achieved when the difference of the total energies of last two consecutive steps is less than $10^{-6} \mathrm{eV}$ and the maximum force allowed on each atom is less than $10^{-2} \mathrm{eV} / \AA$. All configurations studied in this work have also been calculated by

${ }^{a)}$ Electronic mail: ciraci@fen.bilkent.edu.tr. using spin-polarized LDA, which were resulted in nonmagnetic ground state.

Adsorption of a single (isolated) $\mathrm{Li}$ atom on the hollow site of graphene (i.e., $\mathrm{H} 1$ site above the center of hexagon) is modelled by using $(4 \times 4)$ cell of graphene with $1.70 \AA$ minimum $\mathrm{Li}$-graphene distance and with a minimum $\mathrm{Li}-\mathrm{Li}$ distance of $9.77 \AA$, resulting in a binding energy of $E_{L}$ $=1.93 \mathrm{eV}$. Upon adsorption, $\mathrm{Li}$ atom donates part of the charge of its $2 s$ state to the more electronegative carbon atoms at its proximity. Despite the ambiguities in determining the atomic charge, Löwdin analysis estimates that $\mathrm{Li}$ becomes positively charged by donating $q \sim 0.35$ electrons (but $q \sim 0.9$ electrons according to Bader analysis ${ }^{11}$ ). The energy barrier to the diffusion of a single $\mathrm{Li}$ atom on the graphene sheet through top (on top of carbon atoms) and bridge (above the carbon-carbon bond) sites are calculated to be $\Delta Q$ $=0.35$ and $0.14 \mathrm{eV}$, respectively.

Lithium atoms can form a denser coverage on the graphene with a smaller $\mathrm{Li}-\mathrm{Li}$ distance of $4.92 \AA$ forming the $(2 \times 2)$ pattern. Owing to the repulsive interaction between positively charged $\mathrm{Li}$ atoms, the binding energy of $\mathrm{Li}$ atom is smaller than that of the $(4 \times 4)$ cell. For H1 adsorption site [see Fig. 1(a)], the binding energy is calculated to be $E_{L}=0.86 \mathrm{eV}$. The binding energies are relatively smaller at the bridge and top sites, and are 0.58 and $0.56 \mathrm{eV}$, respectively. The binding energy of the second $\mathrm{Li}$ for the double sided adsorption with $\mathrm{H} 1+\mathrm{H} 2$ and $\mathrm{H} 1+\mathrm{H} 3$ configurations described in Fig. 1(a), are $E_{L}=0.82$ and $0.84 \mathrm{eV}$, respectively. The same binding energies for $\mathrm{H} 1+\mathrm{H} 2$, and $\mathrm{H} 1+\mathrm{H} 3$ geometries on the $(4 \times 4)$ cell are relatively larger due to reduced repulsive $\mathrm{Li}-\mathrm{Li}$ interaction, namely $E_{L}=1.40$ and $1.67 \mathrm{eV}$, respectively. The coverage of $\mathrm{Li}$ on the $(2 \times 2)$ cell is $\Theta=12.5 \%$ (i.e., one $\mathrm{Li}$ for every eight carbon atoms) for $\mathrm{H} 1$ geometry and $\Theta=25 \%$ for either $\mathrm{H} 1+\mathrm{H} 2$ or $\mathrm{H} 1+\mathrm{H} 3$ geometries. Metallic charge accumulated between $\mathrm{Li}$ and graphene weakens the interaction between $\mathrm{Li}$ atoms which are adsorbed at different sites of graphene. Further increasing one-sided coverage of $\mathrm{Li}$ to $\Theta=25 \%$ with $\mathrm{H} 1$ geometry (or two-sided coverage to $50 \%$ with $\mathrm{H} 1+\mathrm{H} 2$ or $\mathrm{H} 1+\mathrm{H} 3$ geometries) appears to be impossible due to strong Coulomb repulsion between adsorbed $\mathrm{Li}$ ions and results in a negative binding energy $\left(E_{L} \sim-2.5 \mathrm{eV}\right)$. On the other hand, the total binding energy of all $\mathrm{Li}$ atoms adsorbed on a $(2 \times 2)$ cell 

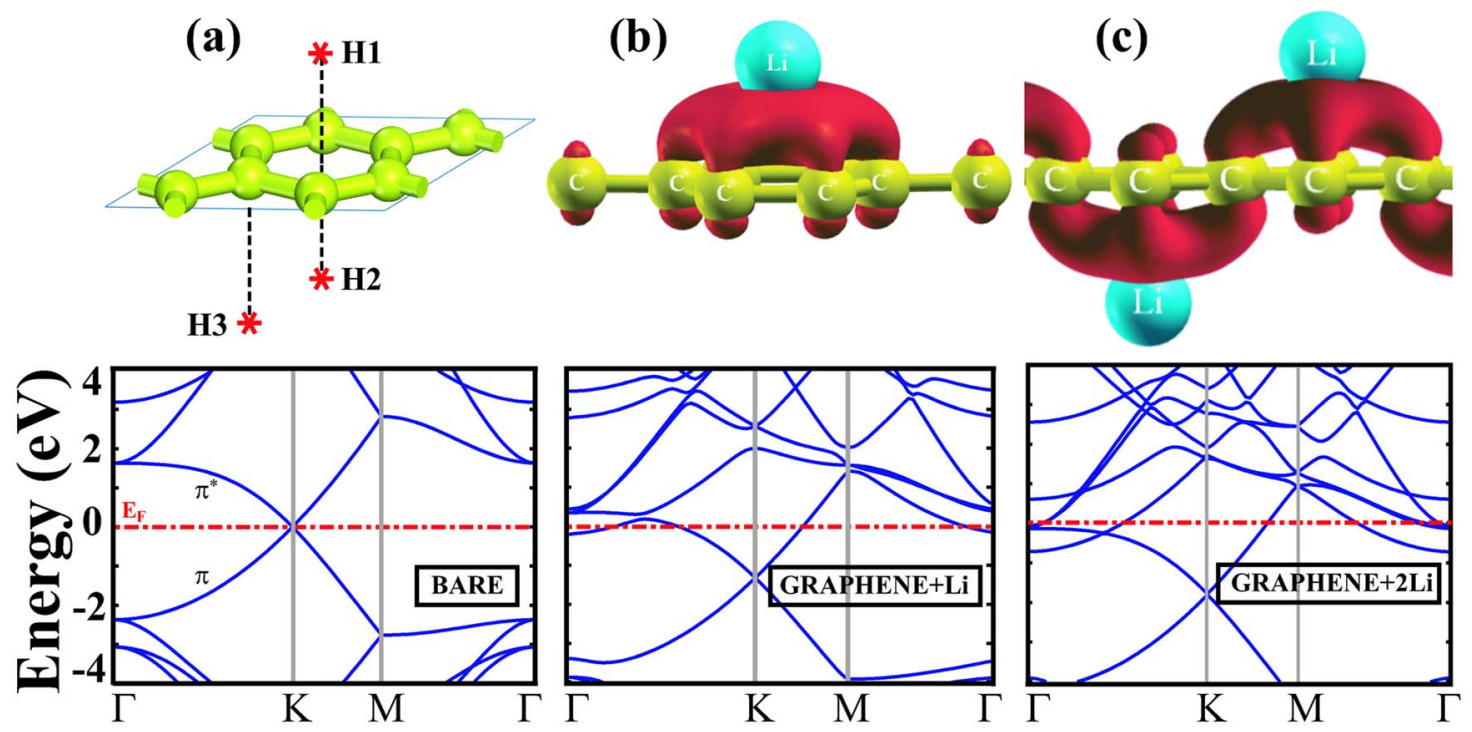

FIG. 1. (Color online) (a) Various adsorption sites H1, H2 and $\mathrm{H} 3$ on the $(2 \times 2)$ cell (top panel) and energy band structure of bare graphene for the same size of cell (bottom panel). (b) Charge accumulation, $\Delta \rho^{+}$, calculated for one Li atom adsorbed to a single side specified as H1 (top) and corresponding band structure. (c) Same as (b) for one Li atom adsorbed to H1 site, second Li adsorbed to H3 site of the $(2 \times 2)$ cell of graphene. Zero of band energy is set to the Fermi energy, $E_{F}$.

with the $\mathrm{H} 1+\mathrm{H} 2(\mathrm{H} 1+\mathrm{H} 3)$ geometry corresponding to $\Theta=25 \%$ is $3.23 \mathrm{eV}(3.12 \mathrm{eV})$ higher than that of $\mathrm{Li}$ atom adsorbed on the $(4 \times 4)$ cell with the same geometry corresponding to $\Theta=6.25 \%$. Hence, since the cluster formation is hindered by the repulsive interaction between the adsorbed ions, a stable and uniform $\mathrm{Li}$ coverage on both sides of graphene up to $\Theta=5 \%$ can be attained.

The charge accumulation and band structure calculated for the $\mathrm{H} 1$ and $\mathrm{H} 1+\mathrm{H} 3$ adsorption geometries are presented in Figs. 1(b) and 1(c), respectively. Isosurface plots of charge accumulation obtained by subtracting charge densities of $\mathrm{Li}$ and bare graphene from that of $\mathrm{Li}$ which is adsorbed to graphene, $\Delta \rho^{+}$, display positive values. As a result of Li adsorption, the charge donated by $\mathrm{Li}$ is accumulated between $\mathrm{Li}$ and graphene and is accommodated by $2 p \pi^{*}$-bonds of carbons. The empty $\pi^{*}$-bands become occupied and eventually get distorted. Occupation of distorted graphene $\pi^{*}$-bands gives rise to the metallization of semimetallic graphene sheets. By controlled Li coverage, one can monitor the position of Fermi energy in the linear region of bands crossing at the $K$ point of the Brillouin zone. Metallization is also important for zigzag and armchair graphene nanoribbons, since both are semiconductors with their energy gaps depending strongly on the widths of these ribbons. ${ }^{12}$ Segments of these ribbons metallized by Li adsorption may be interesting for their electronic and spintronic applications. For example, a junction of two nanoribbons with and without $\mathrm{Li}$ adsorbed segments can serve as a Schottky barrier.

Sodium, a heavier alkali metal, can be bound to graphene with $E_{b}=1.09 \mathrm{eV}$ at $\mathrm{H} 1$ site. However, the energy difference between top, bridge and $\mathrm{H} 1$ sites are minute due to relatively larger radius of $\mathrm{Na}$. Upon adsorption, graphene and graphene nanoribbon are metallized. Nevertheless, $\mathrm{Na}$ is not suitable for hydrogen storage because of its heavier mass and very weak binding to $\mathrm{H}_{2}$ molecules. Two dimensional $\mathrm{BN}$-honeycomb structure, being as a possible alternative to graphene, has very weak binding to $\mathrm{Li}(\sim 0.13 \mathrm{eV})$ and hence it is not suitable for hydrogen storage.
The absorption of $\mathrm{H}_{2}$ molecules by $\mathrm{Li}+$ graphene in $\mathrm{H} 1$, $\mathrm{H} 1+\mathrm{H} 2$, and $\mathrm{H} 1+\mathrm{H} 3$ geometries. A summary of our results about the $\mathrm{H}_{2}$ absorption are presented in Fig. 2. The binding energy of the first absorbed $\mathrm{H}_{2}$, which prefers to be parallel to graphene, is generally small. However, when two or more $\mathrm{H}_{2}$ molecules are absorbed by the same $\mathrm{Li}$ atom, the binding geometry and mechanism change and result in a relatively higher binding energy. All $\mathrm{H}_{2}$ molecules are tilted so that one of two $\mathrm{H}$ atoms of each absorbed $\mathrm{H}_{2}$ molecules becomes relatively closer to the $\mathrm{Li}$ atom. A weak ionic bond forms through a small amount change ( $\geq 0.1$ electrons) transferred from $\mathrm{Li}$ and graphene to nearest $\mathrm{H}$ atoms of absorbed $\mathrm{H}_{2}$ molecules. At the end, $\mathrm{H}$ atoms receiving charge from $\mathrm{Li}$ becomes negatively charged and the covalent $\mathrm{H}_{2}$ bond becomes polarized. Weak ionic bond, attractive Coulomb interaction between positively charged $\mathrm{Li}$ and negatively charged $\mathrm{H}$ and weak van der Waals interaction are responsible for the formation of mixed weak bonding between $\mathrm{H}_{2}$ molecules and $\mathrm{Li}+$ graphene complex. Here the bonding interaction is different from the Dewar-Kubas interaction ${ }^{13}$ found in $\mathrm{H}_{2}-\mathrm{Ti}$ $+\mathrm{C}_{60}$ or carbon nanotube complexes. ${ }^{3}$ As the number of absorbed $\mathrm{H}_{2}, n$, increases, the positive charge on $\mathrm{Li}$ as well as the minimum distance between $\mathrm{H}_{2}$ and Li slightly increases. No matter what the initial geometry of absorbed $\mathrm{H}_{2}$ molecules would be, they are relaxed to the same final geometry presented in Fig. 2 for any given $n$. We found no energy barrier for a $\mathrm{H}_{2}$ molecule approaching the absorbed $\mathrm{H}_{2}$ when $n \leqslant 4$. Note that the dissociative absorption of $\mathrm{H}_{2}$ molecules do not occur in the present system. The energy barrier for the dissociation of $\mathrm{H}_{2}$ near $\mathrm{Li}$ to form $\mathrm{Li}-\mathrm{H}$ bond is $\sim 2 \mathrm{eV}{ }^{14}$ Moreover, dissociation of $\mathrm{H}_{2}$ to form two $\mathrm{C}-\mathrm{H}$ bonds at the graphene surface is energetically unfavorable by $0.7 \mathrm{eV}$.

Maximum number of absorbed $\mathrm{H}_{2}$ per Li atom is four, and the maximum gravimetric density corresponding to $\mathrm{H} 1$ $+\mathrm{H} 2$ geometry at $\Theta=25 \%$ coverage is $g_{d}=12.8 \mathrm{wt} \%$. This is much higher than the limit $\left(g_{d}=6 \mathrm{wt} \%\right)$ set for the feasible $\mathrm{H}_{2}$ storage capacity. Note that only for $n=4, \mathrm{H} 1+\mathrm{H} 2$ absorption geometry has slightly lower energy than $\mathrm{H} 1+\mathrm{H} 3$ geom- 


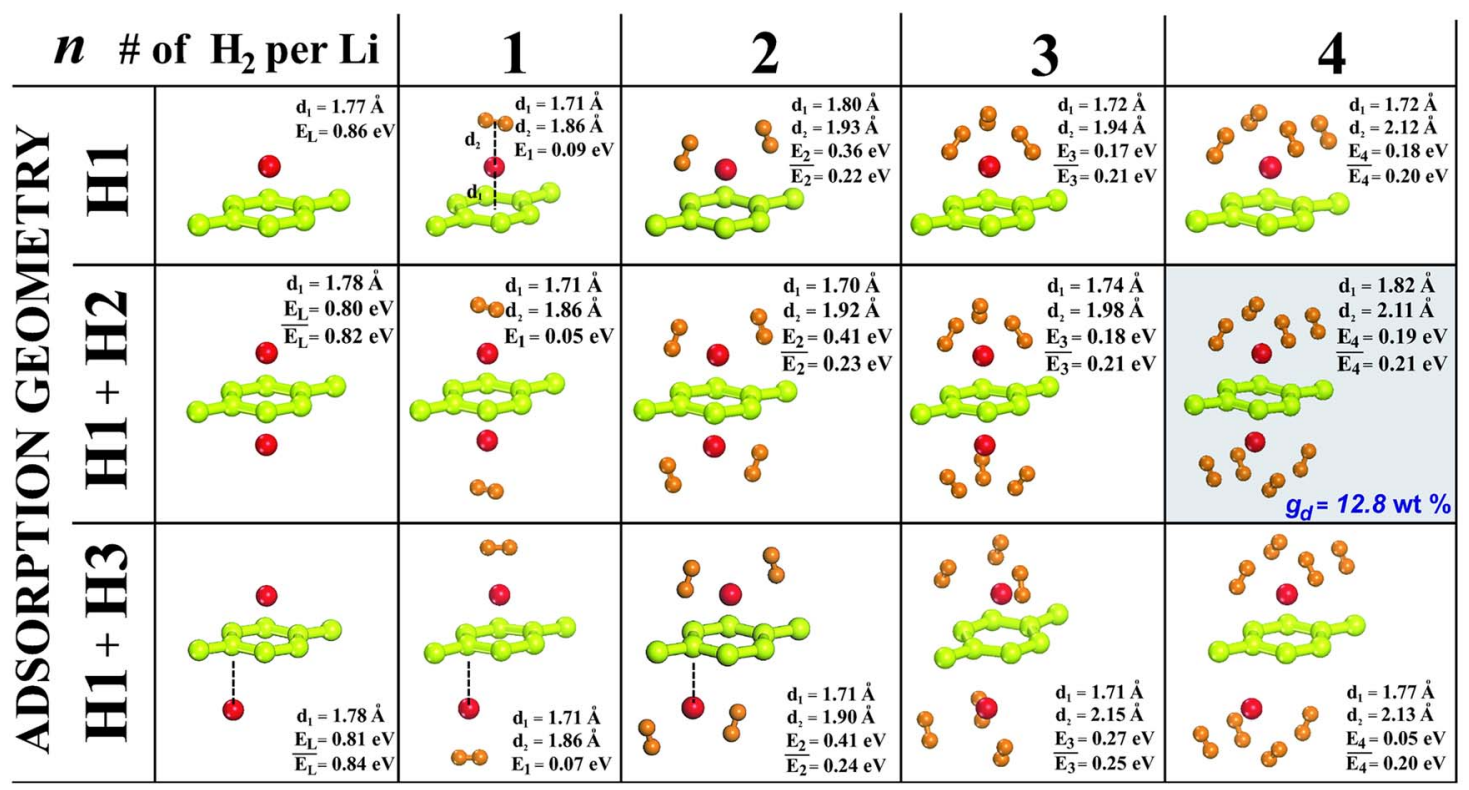

FIG. 2. (Color online) Adsorption sites and energetics of Li adsorbed to the $(2 \times 2)$ cell of graphene and absorption of $\mathrm{H}_{2}$ molecules by $\mathrm{Li}$ atoms. $E_{L}$ is the binding energy of $\mathrm{Li}$ atom adsorbed to $\mathrm{H} 1$ site, which is a minimum energy site. For $\mathrm{H} 1+\mathrm{H} 2$ or $\mathrm{H} 1+\mathrm{H} 3$ configuration corresponding to double sided adsorption, $E_{L}$ is the binding energy of second Li atom and $\bar{E}_{L}$ is the average binding energy. For $\mathrm{H} 1, \mathrm{H} 1+\mathrm{H} 2$ and $\mathrm{H} 1+\mathrm{H} 3$ configurations, $E_{1}$ is the binding energy of the first $\mathrm{H}_{2}$ absorbed by each Li atom; $E_{n}(n=2-4)$ is the binding energy of the last $n$th $\mathrm{H}_{2}$ molecule absorbed by each Li atom, and $\bar{E}_{n}$ is the average binding energy of $n \mathrm{H}_{2}$ molecules absorbed by each Li. Shaded panel indicates the most favorable $\mathrm{H}_{2}$ absorbtion configuration.

etry. This is a remarkable result indicating another application of graphene as a high capacity storage medium. Here $\mathrm{Li}+$ graphene complex is superior to $\mathrm{Ti}+\mathrm{C}_{60}$ or carbon nanotube complexes since $\mathrm{Li}$ is lighter. Even though graphene by itself is stable, ${ }^{15,16}$ more stable form is obtained by $\mathrm{Li}$ adsorption on graphene due to strong Coulomb repulsion between adsorbed $\mathrm{Li}$ atoms. Moreover, $\mathrm{Li}$ covered graphene is resistant to clustering of adsorbed $\mathrm{Li}$ atoms. Earlier, Durgun et $a l^{5}$ has predicted that ethylen+Ti complex can store $\mathrm{H}_{2}$ up to $g_{d}=14.4 \mathrm{wt} \%$ per molecule. Later, their results have been confirmed experimentally. ${ }^{17}$ We believe that hydrogen storage by the Li covered graphene is interesting, since it may not require encapsuling and hence may yield even higher effective $g_{d}$.

In conclusion, two crucial features of $\mathrm{Li}$ covered graphene revealed in this paper may be of technological interest. These are high metallicity and high hydrogen storage capacity of graphene functionalized by $\mathrm{Li}$ atoms. Graphene nanoribbons metallized through adsorbed $\mathrm{Li}$ atoms can be used as interconnects between graphene based spintronic devices. Graphene functionalized by $\mathrm{Li}$ can also serve as a medium of hydrogen storage. As far as efficiency in storage is concerned, graphene may be superior to carbon nanotubes because both its sides are readily utilized. Cell configurations formed by different junctions of graphene ${ }^{18}$ functionalized by $\mathrm{Li}$ atoms are expected to yield higher surface/volume ratio and hence to provide efficient $\mathrm{H}_{2}$ storage in real applications.

This research was supported by the Scientific and Technological Research Council of Turkey under Project No.
TBAG 104536. Part of computational resources have been provided through a grant (20242007) by the National Center for High Performance Computing, Istanbul Technical University.

${ }^{1}$ R. Coontz and B. Hanson, Science 305, 957 (2004).

${ }^{2}$ S. Dag, Y. Ozturk, S. Ciraci, and T. Yildirim, Phys. Rev. B 72, 155404 (2005).

${ }^{3}$ T. Yildirim and S. Ciraci, Phys. Rev. Lett. 94, 175501 (2005).

${ }^{4}$ Y. Zhao, Y.-H. Kim, A. C. Dillon, M. J. Heben, and S. B. Zhang, Phys. Rev. Lett. 94, 155504 (2005).

${ }^{5}$ E. Durgun, S. Ciraci, W. Zhou, and T. Yildirim, Phys. Rev. Lett. 97, 226102 (2006).

${ }^{6}$ K. S. Novosolov and A. K. Geim, Nature (London) 438, 197 (2005).

${ }^{7}$ D. Vanderbilt, Phys. Rev. B 41, 7892 (1990).

${ }^{8}$ M. A. Cordero, L. M. Malina, J. A. Alonso, and L. A. Girifalco, Phys. Rev. B 70, 125422 (2004).

${ }^{9}$ G. Kresse and J. Hafner, Phys. Rev. B 47, R558 (1993).

${ }^{10}$ S. Baroni, A. Del Corso, S. Girancoli, and P. Giannozzi (http:/ www.pwscf.org)

${ }^{11}$ G. Henkelman, A. Arnaldsson, and H. Jonsson, Comput. Mater. Sci. 36, 254-360 (2006).

${ }^{12}$ Y.-W. Son, M. L. Cohen, and S. G. Louie, Phys. Rev. Lett. 97, 216803 (2006).

${ }^{13}$ Metal Dihydrogen and Bond Complexes-Structure, Theory and Reactivity, edited by G. J. Kubas (Kluwer Academics/Plenum, New York, 2001).

${ }^{14}$ Y. L. Zhao, R. Q. Zhang, and R. S. Wang, Chem. Phys. Lett. 398, 62 (2004).

${ }^{15}$ A. K. Geim and K. S. Novoselov, Nat. Mater. 6, 183 (2007).

${ }^{16}$ A. Fasolino, J. H. Los, and M. I. Katsnelson, Nat. Mater. 6, 858 (2007).

${ }^{17}$ A. B. Phillips and B. S. Shivaram, Phys. Rev. Lett. 100, 105505 (2008).

${ }^{18}$ T. Kawai, S. Okada, Y. Miyamoto, and A. Oshiyema, Phys. Rev. B 72, 035428 (2005). 